\title{
Impact of HIV infection and alcohol on cognition: a review
}

\author{
This article was published in the following Dove Press journal: \\ Neurobehavioral HIV Medicine \\ 19 October 2010 \\ Number of times this article has been viewed
}

\section{Ola A Selnes}

Department of Neurology, Division of Cognitive Neuroscience, Johns Hopkins University School of Medicine, Baltimore, MD, USA
Correspondence: Ola A Selnes Department of Neurology, Division of Cognitive Neuroscience, Johns Hopkins University School of Medicine, I620 McElderry St, Reed Hall East-2, Baltimore, MD 21205-1910, USA Email oselnes@jhmi.edu

\begin{abstract}
Both alcohol and HIV (human immunodeficiency virus) can affect the structure and function of the central nervous system. What they have in common is that the white matter of the brain is principally affected, and the direct neuronal injury does not appear to be a consequence of either HIV infection or alcohol use. There is evidence that the cognitive effects of both HIV and alcohol are potentially reversible with treatment or abstinence. There is only limited overlap in terms of the actual brain regions or structures affected, which may account for the observation that the combined effects of alcohol and HIV are largely additive. Although the incidence of alcohol-related Korsakoff amnesia has declined, alcohol use nonetheless is associated with decreased cognitive performance. In the context of HIV infection, very heavy alcohol use in individuals with advanced HIV disease appears to have synergistic effects. Because current studies on the combined effects of alcohol and HIV infection are limited by several issues, including the use of relatively small sample sizes and nonuniform definitions of what constitutes heavy alcohol use, our understanding of the neurological and behavioral complications of this problem still remains incomplete.
\end{abstract}

Keywords: central nervous system, alcohol, cognition, HIV, treatment

"Wine from long habit has become indispensable to my health. Good wine is a necessity of life for me".

Thomas Jefferson (1743-1826)

\section{Introduction}

Wine and other forms of alcohol have long been associated with celebration and enjoyment, but excessive use of alcohol has also been a source of significant morbidity and mortality. Recent statistics from the National Highway Traffic Safety Association suggest that nearly $40 \%$ of all traffic fatalities in the United States were related to alcohol use. ${ }^{1}$ Chronic heavy alcohol use can also be associated with a number of medical comorbidities, including liver disease, digestive problems, cardiovascular disease, and numerous neurological problems. ${ }^{2}$ Some of the more common side effects of high-risk drinking are summarized in Table 1. The risk of alcohol-related medical complications depends not only on the quantity and type of alcohol consumed on a given occasion but also on several other factors, including age, gender, lifestyle, and overall health status. Consequently, there are no specific criteria that can predict how much alcohol can be harmful to an individual's health at a given point in his or her life. The lack of a direct relationship between quantity of alcohol consumed and medical complications is illustrated by the epidemiology of alcohol-related neurological complications. Even though the per capita wine consumption in France is nearly double than that in Australia, the incidence of the alcohol-related 
Table I Common effects of high-risk drinking

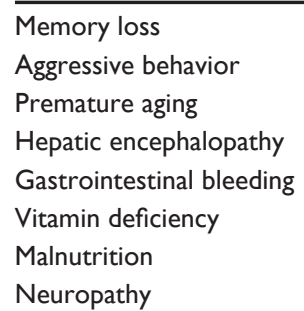

neurological complication of Wernicke-Korsakoff's disease is significantly lower in France than in Australia. ${ }^{3,4}$ The question of how much alcohol is safe has come into focus again since several recent studies have demonstrated modest cardiovascular benefits associated with light to moderate alcohol consumption. ${ }^{5}$

Because alcohol use is often associated with both high-risk sexual behaviors and injection drug use, two of the main routes of HIV (human immunodeficiency virus) transmission, there is some evidence that suggest that individuals who abuse alcohol may be more likely to become HIV-infected than those who do not. ${ }^{6}$ In patients who are already HIV infected, the combination of heavy alcohol use and HIV has been associated with increased medical and psychiatric complications. The majority of individuals infected through injection drug use are also coinfected with hepatitis $\mathrm{C}$. Since both alcohol abuse and hepatitis $\mathrm{C}$ are associated with liver diseases, alcohol use can add to the overall disease burden. Alcohol use has also been associated with decreased compliance with HIV medications, thus, resulting in less favorable treatment outcomes. ${ }^{7}$ It is, therefore, reasonable to expect that decreasing alcohol use in individuals who are already infected with HIV or who are at risk of becoming infected may limit the spread of HIV and its associated comorbidities.

\section{Prevalence of alcohol use and heavy drinking in HIV-infected individuals}

Although there is some consensus that the prevalence of alcohol use is higher among HIV-infected individuals than among the general population, ${ }^{8}$ whether the available prevalence estimates are representative and valid has not been well established. There is also evidence that the prevalence of alcohol use may be changing as the HIV epidemic is evolving. In a study of 3,863 men from the Multicenter AIDS Cohort Study (MACS), it was found that alcohol use declined significantly in the 5-year period between 1984 and 1989, with the greatest degree of decline in the HIV-positive study participants. ${ }^{9}$ Other studies have also concluded that heavy use of alcohol by young gay men is declining although levels of other substance abuse remain high. ${ }^{10}$ More recent estimates of the changes in trends in the use of alcohol in HIV-uninfected individuals suggest an overall decrease in alcohol use and binge drinking but an increase in alcohol use in individuals reporting injection drug use and multiple sex partners. ${ }^{11}$ Most studies of the prevalence of alcohol use have had modest sample sizes or have been limited to specific geographical areas. Some data suggest that prevalence of alcohol use may differ by the route of transmission of HIV, with earlier studies focusing almost exclusively on men who have sex with men. In a 1996 study of 2,864 HIV-infected individuals receiving care, $54 \%$ reported drinking an alcoholic drink during the past 4 weeks, which is similar to the prevalence in the general population. In contrast, $8 \%$ of the subjects reported heavy drinking ( 5 or more drinks on 4 or more days during the past 4 weeks), which is nearly double the prevalence in the general population. The percentage of participants drinking any alcohol was higher among whites, those with a college degree, those with higher incomes, and those exposed to HIV through male-to-male sexual behavior. In contrast, heavy drinking was most common in participants with less education, lower incomes, and with cocaine or heroin use. ${ }^{12}$ The prevalence of heavy drinking did not differ by age.

It is widely assumed that use of alcohol before or during sex will increase the likelihood of high-risk sexual behaviors. During the early years of the HIV epidemic, alcohol use was identified as a specific causative factor for risky sex, and as a result, alcohol use was thought to be a significant risk factor for the spread of HIV infection. ${ }^{13}$ More recent studies have questioned the causal link between alcohol use and highrisk sex behaviors and instead have suggested that men who engage in protected sex when sober also tend to do so when drunk. ${ }^{14,15}$ An alternative explanation for the correlation between alcohol and high-risk sex may be that the personality factors that lead to heavy alcohol use overlap with those that are associated with likelihood of engaging in high-risk sex behaviors even in the absence of alcohol use.

In summary, the prevalence of light or moderate alcohol consumption is similar in HIV-infected populations and the population at large, but heavy alcohol use is higher in $\mathrm{HIV}$-infected individuals. Most of the studies reporting on prevalence of alcohol use in HIV-infected individuals have been limited by small sample sizes; however, although intuitively plausible, the evidence for a direct causal relationship between alcohol use and the risk of engaging in high-risk sexual behaviors has been challenged. 


\section{Effects of alcohol on the brain}

The wide range of behavioral effects of alcohol ingestion is believed to be mediated through the effects of ethanol on several central nervous system (CNS) neurotransmitters. Individual differences in the relative susceptibility of different neurotransmitter systems may explain why the behavioral effects of alcohol range from aggression to euphoria or sedation. ${ }^{1}$ There is evidence that chronic heavy alcohol use is associated with both global and regional brain atrophy in a dose response-related manner. The Atherosclerosis Risk in Communities Study of nearly 2,000 adults and the Cardiovascular Health Study (CHS) of 3,660 adults found magnetic resonance imaging (MRI) evidence of whole-brain atrophy associated with alcohol use. Several variables appear to influence the degree of atrophy associated with alcohol use, including the type of alcoholic beverage used. ${ }^{14}$ There is also evidence that heavy alcohol use is associated with elevated homocysteine levels in plasma, which by itself has been identified as a risk factor for global brain atrophy. ${ }^{16}$

More recent techniques using voxel-based morphometry have allowed a more quantitative assessment of changes in both grey and white matters associated with heavy alcohol use, and have demonstrated decreased volume in several areas, including the thalamus, posterior hippocampus, frontal cortex, pons, and cerebellum. ${ }^{17}$

Largely on the basis of neuroimaging findings, it has been hypothesized that regional atrophy involving the grey matter may be secondary to neuronal loss as a result of neurotoxic effects of alcohol, whereas the more global changes may be related to the loss of white matter (myelin). In contrast, more recent neuropathological studies have not demonstrated any evidence of neuronal loss or other gray matter pathology in the brains of alcoholics. One such study specifically examined the hippocampus, and although overall hippocampal volumes were reduced, this appeared to be related specifically to the white matter rather than to the neuronal loss. ${ }^{18}$

Diffusion tensor imaging (DTI) permits more detailed imaging of the integrity of subcortical white matter tracts, and some recent studies have demonstrated a combined effect of alcohol and HIV infection on degradation of the microstructure of the corpus callosum. The effects were most pronounced in individuals with advanced HIV infection or acquired immunodeficiency syndrome (AIDS). ${ }^{19}$ Although the role of the corpus callosum in higher cognitive functions still remains poorly defined, ${ }^{20}$ the authors reported an association between degradation of corpus callosum microstructure and performance on measures of motor speed. These findings of early structural alterations in the corpus callosum are particularly interesting because very heavy alcohol use by non-HIV-infected individuals can be associated with global demyelination of the fibers of the corpus callosum. ${ }^{21}$

Consistent with the data suggesting mild cardiovascular benefits associated with moderate alcohol use, there is evidence from population studies involving non-HIVinfected subjects that older individuals with light alcohol use have few white matter lesions and silent infarctions in brain on MRI. ${ }^{22}$ Investigators from the CHS reported an inverse U-shaped relationship between the white matter hyperintensities and level of alcohol consumption. Individuals with light to moderate alcohol use had fewer white matter hyperintense signal abnormalities than did those who did not drink any alcohol. ${ }^{23}$ The relationship between white matter hyperintensities and alcohol in the context of HIV infection has not been investigated in detail. In an early study from the MACS, it was found that the frequency of white matter hyperintensities was similar in seronegative and seropositive individuals and there was no association with hypertension or alcohol use. ${ }^{24}$

On a positive note, it has long been known that abstinence from alcohol is associated with improvements in the global alcohol-associated brain atrophy. ${ }^{25}$ By use of a variety of contemporary imaging methods, it has also been demonstrated that there are marked brain microstructural and macrostructural improvements even after relatively short periods of abstinence. ${ }^{26}$

In summary, alcohol produces both structural and physiological changes in the brain. As with the effects of HIV infection on the CNS, alcohol produces the most striking changes in white matter structures. Moreover, neither alcohol nor HIV produces direct neuronal injury of the brain. There is only limited overlap in the brain areas vulnerable to alcohol and those typically affected by HIV (Table 2). The frontal

Table 2 Regional brain involvement by HIV and alcohol

\begin{tabular}{llll}
\hline Brain regions & $\begin{array}{l}\text { Affected by } \\
\text { HIV }\end{array}$ & $\begin{array}{l}\text { Affected by } \\
\text { ETOH }\end{array}$ & Reference \\
\hline Frontal lobes & + & ++ & 29 \\
Temporal lobes & - & - & - \\
Parietal lobes & - & - & - \\
Caudate & ++ & - & 31 \\
Basal ganglia & ++ & - & 32 \\
Hippocampus & - & + & 30 \\
Mammillary bodies & - & ++ & 27 \\
Thalamus & - & + & 75 \\
Cerebellum & - & ++ & 28 \\
Corpus callosum & + & ++ & 19 \\
\hline
\end{tabular}

Abbreviations: HIV, human immunodeficiency virus; ETOH, ethanol. 
lobes, mammillary bodies, and cerebellum are commonly affected by heavy alcohol use ${ }^{27-30}$ but not by HIV infection. Conversely, basal ganglia structures are frequently affected by HIV but not by alcohol use..$^{31,32}$ Other brain structures, such as the corpus callosum, may be vulnerable to both HIV infection and heavy alcohol use. ${ }^{19}$ This limited degree of overlap may explain why the combined effect of moderate alcohol use and HIV in the CNS appears to be predominantly additive. At very high levels of alcohol abuse and more advanced disease stages of HIV, synergistic effects have been reported. ${ }^{8}$ Some of the alcohol-induced central nervous changes are at least partially reversible in individuals who stop drinking alcohol.

\section{Effects of alcohol on non-HIV- infected populations \\ Cognitive effects of chronic alcoholism}

There is ample evidence that chronic heavy alcohol use can have devastating consequences on the CNS. The Wernicke-Korsakoff syndrome, characterized by a profound global amnestic syndrome, is known to occur in a subset of alcoholics. The incidence of Korsakoff syndrome nonetheless does not appear to correlate well with per capita consumption of alcohol, suggesting that factors other than quantity of alcohol consumed also play a role. ${ }^{4}$ Although there appears to have been a decline in the incidence of Korsakoff's syndrome since the introduction of thiaminefortified foods, it nonetheless remains one of the most serious neurological complications of chronic heavy alcohol use. Korsakoff syndrome also occurs in thiamine-deficient nonalcoholic subjects, suggesting that the neurological effect of ethanol by itself is not a key factor in the expression of clinical symptoms. ${ }^{33}$ The incidence of Korsakoff's syndrome has been reported in patients with nutritional deficiencies secondary to bariatric surgery, colectomy, anorexia nervosa, and AIDS. ${ }^{34,35}$ The neuropathology of Wernicke-Korsakoff syndrome has been well described. It typically involves bilateral lesions in structures surrounding the third ventricle, aqueduct, and fourth ventricle. The medial thalamus often shows the most prominent loss of neurons. ${ }^{36}$ Autopsy-confirmed cases of Wernicke-Korsakoff syndrome are not always clinically diagnosed antemortem; an estimated $75 \%-80 \%$ of cases are not identified through routine clinical examination. ${ }^{4}$ This scenario is somewhat similar to that of Alzheimer disease. In community cohorts, an increasing number of elderly patients with Alzheimer type brain pathology at autopsy did not have antemortem clinical signs, symptoms of memory loss, or dementia. ${ }^{37}$
A second, and much less common, neurological syndrome related to heavy alcohol consumption is the condition known as Marchiafava-Bignami disease, which is associated with cerebral and cerebellar atrophy and striking loss of myelin in the corpus callosum..$^{21,38,39}$ Although originally thought to occur only in Italian wine drinkers, Marchiafava-Bignami disease has also been reported in nonalcoholic patients. ${ }^{40}$

\section{Cognitive effects of mild to moderate alcohol use}

In contrast to the devastating global amnestic deficits associated with alcoholic Korsakoff syndrome, the cognitive side effects of less severe forms of alcohol use are more variable. Much of what we know about the cognitive deficits associated with mild to moderate alcohol use is derived from the studies of detoxified alcoholics. Although some studies have reported lower performance in the cognitive domains of executive functioning, visuospatial abilities, and psychomotor speed, ${ }^{41}$ other studies have found the cognitive performance of detoxified alcoholics to be within normal limits. ${ }^{42,43}$ Such discrepancies may be related among other factors to subtle participant selection biases. Some studies have reported that alcoholics who agreed to participate in studies were more likely to be unemployed, have legal problems, and use other drugs than were those not volunteering. ${ }^{44}$ There is also recent evidence that active smoking may play a role in the cognitive performance of detoxified alcoholics. Alcoholics who are active smokers have been found to have worse cross-sectional cognitive performances ${ }^{45}$ and to recover less well over time after periods of abstinence. ${ }^{46}$

Because of the potentially confounding effects of medical, psychiatric, and substance abuse comorbidities associated with chronic alcoholism, some investigators have focused on studying sober social drinkers. The results from such studies have also been somewhat contradictory, with some reporting definite cognitive impairment, whereas others finding no differences between abstinent social drinkers and controls. ${ }^{47}$ The studies that reported significantly worse cognitive performance in heavy social drinkers compared with light social drinkers generally included subjects with a greater number of standard drinks per occasion of drinking. The combined effects of smoking and alcohol use on cognition do not appear to have been systematically examined in social drinkers.

In summary, there is clear evidence that chronic heavy alcohol use may result in significant cognitive impairment, as demonstrated by the global amnestic syndrome seen in patients with Korsakoff syndrome. In contrast, there is little 
evidence to suggest that more moderate levels of alcohol use in the context of adequate nutrition result in inevitable cognitive decline or impairment. A number of different variables, including the duration of alcohol use, the number of drinks per occasion, the nutritional status, and the medical comorbidities, also play a role.

\section{Cognitive implications of HIV infection}

A progressive dementing disorder with lethargy and confusion in HIV-infected patients was recognized early during the HIV epidemic. It was initially thought to be secondary to cytomegalovirus infection of CNS, but later studies demonstrated that rather than being an opportunistic infection, the dementia was due to the invasion of the CNS by the HIV itself. The clinical features of the AIDS dementia complex, also known as HIV dementia or HIV encephalopathy, were first described by Navia et $\mathrm{al}^{48}$ in 1986 . Shortly thereafter, it became clear that many HIV-infected individuals had milder forms of cognitive impairment. Large epidemiological studies (MACS) established that a major predictor of cognitive impairment was the degree of immunosuppression in that individuals with AIDS, or those with CD4 counts below 200 , were at greatest risk of developing cognitive symptoms. Only $5.5 \%$ of asymptomatic individuals were found to have abnormal performance on at least one neuropsychological test, a proportion that did not differ from that seen in seronegative controls. ${ }^{49}$ The advent of antiretroviral therapy with its suppressive effects on systemic viral replication led to a dramatic reduction in the incidence of HIV-associated dementia and milder forms of cognitive impairment. Nonetheless, despite the improvements in current generations of potent antiretroviral medications, the problem of HIV-associated cognitive impairment has not been eliminated. This is in part because many of the newer antiretroviral treatments have poor penetration in the cerebrospinal fluid and brain. In addition, a certain complacency regarding the risks and consequences of HIV infection has developed. It is estimated that of the approximately one million Americans currently infected with HIV, 20\% are unaware that they are HIV positive..$^{50}$ Since these individuals are not protected by antiretroviral therapy, they may be at particularly high risk for CNS replication of the virus and associated cognitive impairment and possible dementia.

\section{Nature of HIV-associated cognitive impairment}

In order to appreciate the effect of alcohol use on cognitive functioning in HIV-infected individuals, it is useful first to characterize the pattern of cognitive symptoms associated with HIV in the absence of alcohol use. Before the introduction of highly active antiretroviral therapies (HAART), the cognitive profile of HIV-associated cognitive impairment was recognized as being generally similar to that of other disorders with predominantly subcortical pathology, including Huntington disease, progressive supranuclear palsy, cerebral autosomal dominant arteriopathy with subcortical infarcts and leukoencephalopathy, and Wilson disease. ${ }^{51,52}$ This subcortical cognitive profile is characterized principally by motor and psychomotor slowing, executive dysfunction, and constructional praxis, ${ }^{53}$ with less pronounced and more variable involvement of other aspects of cognitive performance. Memory is also impaired in patients with more advanced HIV-associated cognitive impairment, but the pattern of the memory impairment is different from that seen in patients with cortical dementia, such as Alzheimer disease. ${ }^{54}$ Patients with HIV-associated dementia typically have impaired acquisition or new learning, but recognition memory tends to be relatively better preserved, even in later stages of the illness. ${ }^{55,56}$

It has been suggested that the pattern of neuropsychological impairment associated with HIV infection has changed since the introduction of HAART ${ }^{57}$ and that there may no longer be a prototypical subcortical signature of cognitive impairment associated with HIV infection. ${ }^{58}$ It is unclear whether this might be related to changes in the localization of the virus in the brain from a predominantly subcortical distribution to a more diffuse involvement or to other factors, such as changes in pattern or magnitude of confounding comorbidities that may be associated with cognitive changes. For example, use of certain drugs of abuse with known cognitive side effects, such as ecstasy, is becoming more widespread among younger HIV-infected individuals. ${ }^{59}$ Consequently, in asymptomatic HIV-infected patients who present with subtle cognitive symptoms or mild cognitive impairment in the absence of the prototypical motor or psychomotor slowing, it is important to consider both HIV-related and non-HIV-related causes of mild cognitive impairment (Table 3).

\section{Memory deficits in HIV}

Consistent with the predominantly fronto subcortical pattern of cognitive involvement with HIV infection, memory is often relatively mildly impaired. In clinically asymptomatic, nonimmuno compromised HIV-infected individuals, new learning and free recall are generally within normal limits. ${ }^{60}$ Patients with more advanced disease and immune 
Table 3 Non-HIV-related causes of mild cognitive impairment

Sleep disturbance (eg, sleep apnea)

Psychoactive medication side effects

Stress/anxiety

Alcohol/substance use

Closed head injury

Small vessel ischemic disease

Vitamin $\mathrm{B}_{12}$ deficiency

Thyroid disease

Dehydration

Malnutrition

suppression may have mild to moderate deficits in both new learning and recall. ${ }^{61}$

Recognition memory has repeatedly been shown to be relatively intact, even in patients with more advanced stages of HIV infection. This is important for several reasons. First of all, it suggests that the cognitive processes involved in registration or new learning are working, but the processes involved in retrieval or free recall may be impaired. Second, this pattern is consistent with the involvement of subcortical white matter tracts rather than the primary hippocampal involvement seen in cortical dementias, such as Alzheimer disease. Third, from a clinical point of view, patients whose recognition memory is comparatively preserved typically can manage their everyday affairs in a relatively competent fashion, despite often having significant memory complaints.

One aspect of memory that is believed to be particularly relevant to maintain independence in everyday living is prospective memory. It refers to the ability to remember to carry out specific tasks, such as taking one's medication, at some time in the future. At different stages of HIV infection, it has been shown that performance on measures of prospective memory is associated with the degree of independence in activities of daily living. ${ }^{62}$ It is unclear whether prospective memory deficits are specifically related to CNS effects of HIV infection or whether they are explained by other factors such as concomitant alcohol and drug use. ${ }^{63}$

When comparing memory performance between seropositive and seronegative individuals, it is often difficult to control for the plethora of factors that can influence memory performance. These include common issues like sleep disturbance and/or use of sleep medications, fatigue, nutritional issues, and previous head injury. There are also numerous sociodemographic issues to consider, including learning disabilities, attention-deficit disorder, and low IQ. Consequently, a direct causal link between memory impairment and specific effects of the virus on the nervous system is often difficult to establish.

\section{Effects of alcohol use on cognitive functions in HIV-infected individuals}

Several studies have examined the effect of alcohol use on neuropsychological performance in HIV-infected individuals. Most of these studies have included HIV-negative and HIV-positive subjects with and without history of heavy alcohol use. It makes intuitive sense that if HIV infection by itself may produce cognitive impairment, use of alcohol in the context of HIV infection should result in even more pronounced cognitive changes, and most studies have concluded that heavy alcohol use interacts with HIV infection to produce more significant cognitive deficits.

Green et $\mathrm{al}^{64}$ compared four groups of gay or bisexual men on a battery of neuropsychological tests. The study participants were stratified on the basis of a positive or negative history of previous alcohol use. Subjects with a history of alcohol use had worse performance in verbal reasoning, auditory information processing, reaction time, and verbal IQ. Main effects of HIV serostatus were observed in the domains of new learning, delayed recall, and motor speed (Grooved Pegboard). These findings are somewhat surprising since ethanol has traditionally been associated with worse performance in areas of memory and executive functions. The authors found no differences in neuropsychological performance between HIV-negative participants with or without a history of alcohol use. In contrast, the HIV-positive participants with a history of alcohol abuse showed worse performance than did those without a history of alcohol abuse in verbal IQ, verbal reasoning, and reaction time. The authors conclude that in HIV-positive subjects, a history of alcohol abuse manifests as lower cognitive performance in some cognitive domains. Although this is a plausible interpretation, this study has several limitations, and a direct causal relationship between the lower cognitive performance and history of alcohol abuse cannot definitely be established. First, the sample was relatively small in all groups. Second, the estimates of current alcohol use were based on self-report, with the somewhat paradoxical finding that the subjects with past history of alcohol use reported virtually no current alcohol use and the ones without a past history reported an average of $50 \mathrm{~g}$ of alcohol per week. The study participants with a history of alcohol abuse had significantly lower verbal IQ scores, and since there is no evidence to suggest that alcohol use causes a reduction 
in verbal IQ, it is possible that some of the differences in cognitive performance in this sample could have been due to the preexisting differences in IQ rather than differences in history of alcohol abuse. The study also did not report any of the comorbidities typically associated with a history of alcohol abuse, such as smoking.

In a large community sample of 497 African-American men, Durvasula et $\mathrm{al}^{65}$ examined the effect of alcohol and serostatus on neurocognitive performance. They found that their seropositive participants with the heaviest alcohol use had lower performance in some cognitive domains compared with seropositives who were drinking less alcohol and also compared with seronegative heavy drinkers. For that reason, they concluded that heavy alcohol use acts synergistically with serostatus to produce worse cognitive performance. Interestingly, the negative effects of alcohol use were observed only in the domains typically affected by HIV (motor and psychomotor speed) and not in domains typically affected by alcohol alone (memory and executive functions). This study illustrates many of the difficulties associated with recruiting groups of participants who differ in their degree and duration of alcohol use but who are otherwise comparable in terms of demographic and other variables that might be expected to influence the performance on standardized cognitive tests. Heavy alcohol use can be associated with a number of medical comorbidities that may influence the pattern and degree of neurocognitive abnormalities, ${ }^{66}$ and it is not surprising that the study by Durvasula et $\mathrm{al}^{65}$ showed that the subjects with the highest level of alcohol use also were significantly more likely to have had a history of closed head injury and loss of consciousness than were the subjects with more modest or no alcohol use. There was also no adjustment for the level of premorbid IQ in this study, which is known to have significant effects on overall levels of neurocognitive performance.

In another community-based study in the San Francisco Bay area, Rothlind et $\mathrm{al}^{8}$ compared neuropsychological test performance in HIV-seronegative and HIV-seropositive volunteers stratified by the level of light vs heavy alcohol use. A comprehensive battery of neuropsychological tests covered working memory, verbal and visual memory, visuoconstruction, and executive and motor functions. Measures of IQ were included as covariates. They found that heavy drinking was associated with worse performance in the areas of working memory and balance and executive functions. Compared with the seronegative controls, HIV-positive participants had lower scores in the domains of processing speed and auditory working memory. HIV-positive participants with lower levels of viral burden had better performance in measures of speed of processing, but the effect of alcohol on cognitive performance did not differ significantly by viral load. Moreover, HIV and chronic heavy drinking did not show any clear synergistic effects. To investigate the effects of very heavy drinking, this group was further stratified into currently heavy drinkers $(n=61$, defined as $<6$ drinks per occasion during the past week) and currently very heavy drinkers $(n=42$, defined as $>6$ drinks per occasion during the past week). The results of this analysis were consistent with synergistic adverse effects of very heavy alcohol use in the cognitive domains of motor and visuomotor functioning, suggesting concurrent very heavy alcohol use and HIV disease may result in lower cognitive performance than with either condition alone. In addition, seropositive participants with viral loads below 400 copies/mL did not differ from the seronegative participants in level of performance on the neurocognitive tests, reinforcing the view that immune status is an important predictor of the clinical expression of cognitive effects associated with HIV infection.

There is, thus, considerable evidence that active heavy alcohol use in the context of HIV infection can adversely affect cognitive performance. In the setting of very heavy active alcohol use, there is further evidence of a synergistic effect of alcohol. Currently, there are no published studies regarding the incidence of HIV dementia and alcohol use. Because very heavy alcohol use is typically confounded with a number of lifestyle issues and medical comorbidities, conclusive evidence that the adverse effects of alcohol use on cognition are mediated specifically by neurotoxic effects of alcohol is lacking. From a clinical view point, the issue of causality is somewhat academic since ultimately the recommendation must be that HIV-infected patients should be made aware of the risk of cognitive side effects of alcohol and counseled to avoid heavy alcohol use.

Although complete abstinence from alcohol may seem like the safest recommendation for any patient infected with HIV, this has to be tempered somewhat by the evidence that modest, social use of alcohol may infer mild cardio protective effects. ${ }^{67}$ Thanks to effective antiretroviral therapies, HIV-infected individuals are now living a much longer life and the risk of developing complications from cerebrovascular disease increases significantly with advancing age. Recent evidence suggests that measures of cardiovascular risk factors are better predictors of cognitive performance in older HIV-infected subjects than HIV serostatus by itself. ${ }^{68}$ 
For that reason, at least in HIV-positive individuals at risk of age-related cerebrovascular disease, it is appropriate to advise that modest use of some forms of alcohol, such as red wine, in combination with optimal management of other modifiable risk factors for cerebrovascular disease may be associated with mild risk reduction for cognitive complications of cerebrovascular disease. For a variety of reasons, the risk-to-benefit ratio of such a recommendation needs to be done on a patient-by-patient basis.

Beyond the cognitive issues, there is also the risk that heavy alcohol use may lead to further suppression of the immune system and, in turn, increase the rate of progression of the disease. ${ }^{69}$ In addition, heavy alcohol use may result in decreased adherence to antiretroviral treatment regimens and, thus, further accelerate the disease process. There is also some evidence that HAART may be less effective in individuals who are heavy alcohol users. ${ }^{70,71}$ Less is known about alcohol abuse and risk of discontinuing antiretroviral therapy. One study reported that in individuals with heavy alcohol use, symptoms of depression rather than the level of alcohol use predicted the risk of stopping antiretroviral therapy. ${ }^{72}$

\section{Future directions}

The study of the effects of alcohol on cognition and behavior is complicated because of a number of issues. One of the fundamental problems is the quantification of the amount of alcohol consumed. This is most often accomplished through self-report, which has known limitations. The use of terms like abstinent, social drinker, heavy drinker, and binge drinker has not been standardized. ${ }^{73}$ Since there is evidence that heavy frequent drinking may be particularly damaging to the nervous system, the definition of what constitutes a binge drinker should be clarified. Binge drinking has traditionally been defined as five or more drinks on any one occasion, but a recent study examining EEG changes associated with heavy alcohol use defined high-binge drinkers as having 10 or more drinks in a 2-hour period. Because both alcoholism and HIV infection are associated with a variety of comorbidities, including hepatitis $\mathrm{C}$ virus infection, nutritional deficiencies, injection drug use, and sleep disorders, the choice of wellmatched control groups with a similar pattern of comorbidities can be very challenging. More sensitive methods for measuring the effects of alcohol on the nervous system may also be important. Newer imaging techniques, such as DTI, have demonstrated regional white matter changes associated with heavy alcohol use. In a recent functional MRI study, investigators reported that binge drinkers had reduced hippocampal activation than demographically similar nondrinkers during a paired associates learning task. ${ }^{74}$ To date, the majority of studies have been cross-sectional studies, and longitudinal studies to examine the effects of alcohol use on HIV disease progression over time could add significantly to our understanding of the implications of alcohol use in the context of HIV infection.

\section{Acknowledgments}

This study was supported by grants MH075673, AI035042, DA004334, and DA 12568 from the National Institutes of Health, Bethesda, Maryland. The author is grateful to Dr Pamela Tallalay for her help with editing this manuscript.

\section{Disclosure}

The author reports no conflicts of interest in this work.

\section{References}

1. Chou SP, Dawson DA, Stinson FS, et al. The prevalence of drinking and driving in the United States, 2001-2002: results from the national epidemiological survey on alcohol and related conditions. Drug Alcohol Depend. 2006;83:137-146.

2. Mannelli P, Pae CU. Medical comorbidity and alcohol dependence. Curr Psychiatry Rep. 2007;9:217-224.

3. Harper C, Fornes P, Duyckaerts C, Lecomte D, Hauw JJ. An international perspective on the prevalence of the Wernicke-Korsakoff syndrome. Metab Brain Dis. 1995;10:17-24.

4. Sechi G, Serra A. Wernicke's encephalopathy: new clinical settings and recent advances in diagnosis and management. Lancet Neurol. 2007;6: 442-455.

5. Corder R, Mullen W, Khan NQ, et al. Oenology: red wine procyanidins and vascular health. Nature. 2006;444:566.

6. Baliunas D, Rehm J, Irving H, Shuper P. Alcohol consumption and risk of incident human immunodeficiency virus infection: a meta-analysis. Int J Public Health. 2010;55:159-166.

7. Lucas GM, Gebo KA, Chaisson RE, Moore RD. Longitudinal assessment of the effects of drug and alcohol abuse on HIV-1 treatment outcomes in an urban clinic. AIDS. 2002;16:767-774.

8. Rothlind JC, Greenfield TM, Bruce AV, et al. Heavy alcohol consumption in individuals with HIV infection: effects on neuropsychological performance. J Int Neuropsychol Soc. 2005;11:70-83.

9. Sullivan PE, Becker JT, Dew MA, et al. Longitudinal trends in the use of Illicit drugs and alcohol in the Multicenter AIDS Cohort Study. Addict Res Theory. 1993;1:279-290.

10. Crosby GM, Stall RD, Paul JP, Barrett DC. Alcohol and drug use patterns have declined between generations of younger gay-bisexual men in San Francisco. Drug Alcohol Depend. 1998;52:177-182.

11. Sander PM, Cole SR, Ostrow DG, Mehta SH, Kirk GD. Determinants of alcohol consumption in HIV-uninfected injection drug users. Drug Alcohol Depend. 2010;111(1-2):173-176.

12. Galvan FH, Bing EG, Fleishman JA, et al. The prevalence of alcohol consumption and heavy drinking among people with HIV in the United States: results from the HIV Cost and Services Utilization Study. J Stud Alcohol. 2002;63:179-186.

13. Hirshfield S, Remien RH, Humberstone M, Walavalkar I, Chiasson MA. Substance use and high-risk sex among men who have sex with men: a national online study in the USA. AIDS Care. 2004;16:1036-1047.

14. Weinhardt LS, Carey MP. Does alcohol lead to sexual risk behavior? Findings from event-level research. Annu Rev Sex Res. 2000;11: $125-157$. 
15. Howe CJ, Sander PM, Plankey MW, Cole SR. Effects of time-varying exposures adjusting for time-varying confounders: the case of alcohol consumption and risk of incident human immunodeficiency virus infection. Int J Public Health. 2010;55:227-228.

16. Sachdev PS, Valenzuela M, Wang XL, Looi JC, Brodaty H. Relationship between plasma homocysteine levels and brain atrophy in healthy elderly individuals. Neurology. 2002;58:1539-1541.

17. Wilhelm J, Frieling H, von AN, Hillemacher T, Kornhuber J, Bleich S. Apolipoprotein E polymorphism, homocysteine serum levels and hippocampal volume in patients with alcoholism: an investigation of a geneenvironment interaction. Pharmacogenomics J. 2008;8:117-121.

18. Harding AJ, Wong A, Svoboda M, Kril JJ, Halliday GM. Chronic alcohol consumption does not cause hippocampal neuron loss in humans. Hippocampus. 1997;7:78-87.

19. Pfefferbaum A, Rosenbloom MJ, Adalsteinsson E, Sullivan EV. Diffusion tensor imaging with quantitative fibre tracking in HIV infection and alcoholism comorbidity: synergistic white matter damage. Brain. 2007;130:48-64.

20. Selnes OA. The corpus callosum: some anatomical and functional considerations with special reference to language. Brain Lang. 1974;1: 111-139.

21. Bano S, Mehra S, Yadav SN, Chaudhary V. Marchiafava-Bignami disease: role of neuroimaging in the diagnosis and management of acute disease. Neurol India. 2009;57:649-652.

22. Den Heijer T, Vermeer SE, van Dijk EJ, et al. Alcohol intake in relation to brain magnetic resonance imaging findings in older persons without dementia. Am J Clin Nutr. 2004;80:992-997.

23. Mukamal KJ, Longstreth WT Jr, Mittleman MA, Crum RM, Siscovick DS. Alcohol consumption and subclinical findings on magnetic resonance imaging of the brain in older adults: the cardiovascular health study. Stroke. 2001;32:1939-1946.

24. McArthur JC, Kumar AJ, Johnson DW, et al. Incidental white matter hyperintensities on magnetic resonance imaging in HIV-1 infection. Multicenter AIDS Cohort Study. J Acquir Immune Defic Syndr. 1990;3: 252-259.

25. Carlen PL, Penn RD, Fornazzari L, Bennett J, Wilkinson DA, Wortzman G. Computerized tomographic scan assessment of alcoholic brain damage and its potential reversibility. Alcohol Clin Exp Res. 1986; 10:226-232.

26. Gazdzinski S, Durazzo TC, Mon A, Yeh PH, Meyerhoff DJ. Cerebral white matter recovery in abstinent alcoholics - a multimodality magnetic resonance study. Brain. 2010;133:1043-1053.

27. Sullivan EV, Pfefferbaum A. Neuroimaging of the Wernicke-Korsakoff syndrome. Alcohol Alcohol. 2009;44:155-165.

28. Baker KG, Harding AJ, Halliday GM, Kril JJ, Harper CG. Neuronal loss in functional zones of the cerebellum of chronic alcoholics with and without Wernicke's encephalopathy. Neuroscience. 1999;91: 429-438.

29. Mechtcheriakov S, Brenneis C, Egger K, Koppelstaetter F, Schocke M Marksteiner J. A widespread distinct pattern of cerebral atrophy in patients with alcohol addiction revealed by voxel-based morphometry. J Neurol Neurosurg Psychiatry. 2007;78:610-614.

30. Harper C. The neuropathology of alcohol-specific brain damage, or does alcohol damage the brain? J Neuropathol Exp Neurol. 1998;57: 101-110.

31. Ances BM, Roc AC, Wang J, et al. Caudate blood flow and volume are reduced in HIV+ neurocognitively impaired patients. Neurology. 2006;66:862-866.

32. Ances BM, Sisti D, Vaida F, et al. Resting cerebral blood flow: a potential biomarker of the effects of HIV in the brain. Neurology. 2009;73:702-708

33. Becker JT, Furman JMR, Panisset M, Smith C. Characteristics of the memory loss of a patient with Wernicke-Korsakoff's syndrome without alcoholism. Neuropsychologia. 1990;28:171-179.

34. Butterworth RF, Gaudreau C, Vincelette J, Bourgault AM, Lamothe F, Nutini AM. Thiamine deficiency and Wernicke's encephalopathy in AIDS. Metab Brain Dis. 1991;6:207-212.
35. Schwenk J, Gosztonyi G, Thierauf P, Iglesias J, Langer E. Wernicke's encephalopathy in two patients with acquired immunodeficiency syndrome. J Neurol. 1990;237:445-447.

36. Donnino MW, Vega J, Miller J, Walsh M. Myths and misconceptions of Wernicke's encephalopathy: what every emergency physician should know. Ann Emerg Med. 2007;50:715-721.

37. Bennett DA, Schneider JA, Arvanitakis Z, et al. Neuropathology of older persons without cognitive impairment from two community-based studies. Neurology. 2006;66:1837-1844.

38. Kim MJ, Kim JK, Yoo BG, Kim KS, Jo YD. Acute MarchiafavaBignami disease with widespread callosal and cortical lesions. J Korean Med Sci. 2007;22:908-911.

39. Raina S, Mahesh DM, Mahajan J, Kaushal SS, Gupta D, Dhiman DS. Marchiafava-Bignami disease. J Assoc Physicians India. 2008;56: 633-635.

40. Murthy SB, Jawaid A, Bock JE, Qureshi SU, Schulz PE. MarchiafavaBignami disease (MBD) in a nonalcoholic patient: a case report. Can J Neurol Sci. 2010;37:138-140.

41. Sullivan EV, Rosenbloom MJ, Pfefferbaum A. Pattern of motor and cognitive deficits in detoxified alcoholic men. Alcohol Clin Exp Res. 2000;24:611-621.

42. Grant I, Adams K, Reed R. Normal neuropsychological abilities of alcoholic men in their late thirties. Am J Psychiatry. 1979;136:1263-1269.

43. Krabbendam L, Visser PJ, Derix MM, et al. Normal cognitive performance in patients with chronic alcoholism in contrast to patients with Korsakoff's syndrome. J Neuropsychiatry Clin Neurosci. 2000;12: 44-50.

44. Rychtarik RG, McGillicuddy NB, Connors GJ, Whitney RB. Participant selection biases in a randomized clinical trial of alcoholism treatment settings and intensities. Alcohol Clin Exp Res. 1998;22:969-973.

45. Durazzo TC, Rothlind JC, Gazdzinski S, Banys P, Meyerhoff DJ. A comparison of neurocognitive function in nonsmoking and chronically smoking short-term abstinent alcoholics. Alcohol. 2006;39:1-11.

46. Durazzo TC, Rothlind JC, Gazdzinski S, Banys P, Meyerhoff DJ. Chronic smoking is associated with differential neurocognitive recovery in abstinent alcoholic patients: a preliminary investigation. Alcohol Clin Exp Res. 2007;31:1114-1127.

47. Parsons OA, Nixon SJ. Cognitive functioning in sober social drinkers: a review of the research since 1986. J Stud Alcohol. 1998;59:180-190.

48. Navia BA, Jordan BD, Price RW. The AIDS dementia complex: I. Clinical features. Ann Neurol. 1986;19:517-524.

49. Miller EN, Selnes OA, McArthur JC, et al. Neuropsychological performance in HIV-1 infected homosexual men: the Multicenter AIDS Cohort Study (MACS). Neurology. 1990;40:197-203.

50. El-Sadr WM, Mayer KH, Hodder SL. AIDS in America - forgotten but not gone. $N$ Engl J Med. 2010;362:967-970.

51. Cummings JL. Subcortical Dementia. New York: Oxford University Press; 1990.

52. Dichgans M. CADASIL: a monogenic condition causing stroke and subcortical vascular dementia. Cerebrovasc Dis. 2002;13 Suppl 2:37-41.

53. Suarez SV, Stankoff B, Conquy L, et al. Similar subcortical pattern of cognitive impairment in AIDS patients with and without dementia. Eur J Neurol. 2000;7:151-158.

54. Graham NL, Emery T, Hodges JR. Distinctive cognitive profiles in Alzheimer's disease and subcortical vascular dementia. J Neurol Neurosurg Psychiatry. 2004;75:61-71.

55. Becker JT, Caldararo R, Lopez OL, Dew MA, Dorst SK, Banks G. Qualitative features of the memory deficit associated with HIV infection and AIDS: cross-validation of a discriminant function classification scheme. J Clin Exp Neuropsychol. 1995;17:134-142.

56. Selnes OA. Memory loss in persons with HIV/AIDS: assessment and strategies for coping. AIDS Read. 2005;15:289-292, 294.

57. Cysique LA, Maruff P, Brew BJ. Prevalence and pattern of neuropsychological impairment in human immunodeficiency virus-infected/ acquired immunodeficiency syndrome (HIV/AIDS) patients across pre- and post-highly active antiretroviral therapy eras: a combined study of two cohorts. J Neurovirol. 2004;10:350-357. 
58. Dawes S, Suarez P, Casey CY, et al. Variable patterns of neuropsychological performance in HIV-1 infection. J Clin Exp Neuropsychol. 2008;30:613-626.

59. Nulsen CE, Fox AM, Hammond GR. Differential effects of ecstasy on short-term and working memory: a meta-analysis. Neuropsychol Rev. 2010;20:21-32.

60. Castelo JM, Sherman SJ, Courtney MG, Melrose RJ, Stern CE. Altered hippocampal-prefrontal activation in HIV patients during episodic memory encoding. Neurology. 2006;66:1688-1695.

61. Odiase FE, Ogunrin OA, Ogunniyi AA. Memory performance in HIV/ AIDS - a prospective case control study. Can J Neurol Sci. 2007; 34:154-159.

62. Woods SP, Ludicello JE, Moran LM, Carey CL, Dawson MS, Grant I. HIV-associated prospective memory impairment increases risk of dependence in everyday functioning. Neuropsychology. 2008;22:110-117.

63. Hadjiefthyvoulou F, Fisk JE, Montgomery C, Bridges NJ. Everyday and prospective memory deficits in ecstasy/polydrug users. J Psychopharmacol. 2010

64. Green JE, Saveanu RV, Bornstein RA. The effect of previous alcohol abuse on cognitive function in HIV infection. Am J Psychiatry. 2004; 161:249-254.

65. Durvasula RS, Myers HF, Mason K, Hinkin C. Relationship between alcohol use/abuse, HIV infection and neuropsychological performance in African American men. J Clin Exp Neuropsychol. 2006;28:383-404.

66. Durazzo TC, Rothlind JC, Gazdzinski S, Meyerhoff DJ. The relationships of sociodemographic factors, medical, psychiatric, and substancemisuse co-morbidities to neurocognition in short-term abstinent alcohol-dependent individuals. Alcohol. 2008;42:439-449.
67. Collins MA, Neafsey EJ, Mukamal KJ, et al. Alcohol in moderation, cardioprotection, and neuroprotection: epidemiological considerations and mechanistic studies. Alcohol Clin Exp Res. 2009;33:206-219.

68. Becker JT, Kingsley L, Mullen J, et al. Vascular risk factors, HIV serostatus, and cognitive dysfunction in gay and bisexual men. Neurology. 2009;73:1292-1299.

69. Shuper PA, Neuman M, Kanteres F, Baliunas D, Joharchi N, Rehm J. Causal considerations on alcohol and HIV/AIDS - a systematic review. Alcohol Alcohol. 2010;45:159-166.

70. Flexner CW, Cargill VA, Sinclair J, Kresina TF, Cheever L. Alcohol use can result in enhanced drug metabolism in HIV pharmacotherapy. AIDS Patient Care STDS. 2001;15:57-58.

71. Cooper CL, Cameron DW. Effect of alcohol use and highly active antiretroviral therapy on plasma levels of hepatitis $\mathrm{C}$ virus (HCV) in patients coinfected with HIV and HCV. Clin Infect Dis. 2005;41 Suppl 1: S105-S109.

72. Kim TW, Kertesz SG, Horton NJ, Tibbetts N, Samet JH. Episodic homelessness and health care utilization in a prospective cohort of HIV-infected persons with alcohol problems. BMC Health Serv Res. 2006;6:19.

73. Glassman TJ. Alcohol measures and terms: a perfect storm for chronic confusion. J Am Coll Health. 2010;58:397-399.

74. Schweinsburg AD, McQueeny T, Nagel BJ, Eyler LT, Tapert SF. A preliminary study of functional magnetic resonance imaging response during verbal encoding among adolescent binge drinkers. Alcohol. 2010;44:111-117.
Neurobehavioral HIV Medicine

\section{Publish your work in this journal}

Neurobehavioral HIV Medicine is an international, peer-reviewed, open access journal focusing on advances in research in HIV/ AIDS, with specific reference to the neurological, psychiatric and behavioral consequences of the disease, concomitant infections and specific antiretroviral therapy. The manuscript

\section{Dovepress}

management system is completely online and includes a very quick and fair peer-review system, which is all easy to use Visit http://www.dovepress.com/testimonials.php to read real quotes from published authors. 